\title{
ISOLATED EYE LID MYOTONIA IN A CHILD
}

\author{
Riaz Ahmed Syed, Mai Abou Seoud. \\ Department of Pediatric Neurology, King Fahad Armed Forces Hospital, Jeddah, Saudi Arabia.
}

\begin{abstract}
A seven-year-old girl presented with inability to open her eyelids after instilling natural tear eye drops. Mother felt that the child was deliberately not opening the eyes. Electromyography (EMG) showed Needle EMG (electromyography) revealed short runs of myotonic discharges in the form repetitive muscle fibre after-discharges on tapping the muscle belly (dive-bomber phenomenon) of left biceps brachie, brachioradialis and rectus femoris suggestive of myotonia. She was diagnosed to have isolated eyelid myotonia.
\end{abstract}

\author{
ARTICLE HISTORY \\ Received 18 August 2020 \\ Accepted 5 December 2020
}

\section{KEYWORDS}

\section{Introduction}

The word myotonia denotes a symptom of delayed relaxation of skeletal muscles after voluntary contraction. ${ }^{1}$ It can affect any muscle group and repeated efforts are needed to relax those contracted muscles. Myotonia is a major symptom in myotonic dystrophy, myotonia congenita and paramyotonia congenita. ${ }^{1}$ Non dystrophic myotonias (NDM) have distinct clinical phenotypes with different ranges of severity from severe neonatal myotonia to mild types of later onset forms. ${ }^{1}$ Genetic mutations of voltage gated sodium and chloride channels are causative in most of such patients. ${ }^{2}$ Despite significant advances in the understanding the pathophysiology of this disorders in terms of clinical and molecular genetics, little is known about the long-term outcome and specific treatment modalities. Isolated eyelid myotonia is a form of a benign disorder, presumed to be due to sodium channel disorder of the eyelid muscles. ${ }^{3}$ We present a seven-year-old girl with inability to open her eyelids after instilling eye drops. It was mistaken for voluntary refusal by the child to open her eyes. Electromyography (EMG) was suggestive of localized myotonia.

\section{Case Report}

A seven-year-old girl was brought to the general pediatric clinic with the complaints from the mother that she refuses to open her eyes for some time, after applying eye drops (natural tears) which she was prescribed by general practitioner (GP) for gritty eyes. On questioning further, the girl attributed this as her disability to open immediately rather than voluntary refusal. She practically had to retract the eye lids with her fingers to open them as she could not do by herself. She also said that she encountered similar problem while sneezing repeatedly. When examined in the neurology clinic, no myotonia was seen at rest or on voluntary tapping the muscles of the body. Even

Address for Correspondence: Dr Riaz Ahmed MD DM DCH FRCPCH FRCP(I), Sr Consultant and Head of Pediatric Neurology, King Fahad Armed Forces Hospital, Jeddah, Saudi Arabia.

Email: nodisability@yahoo.com

(C)2021 Pediatric Oncall on tight closure of eyelids, myotonia could not be induced until the mother suggested instilling a drop of natural tear eye-drop. There was no positive lid lag and retraction sign. Sneezing was induced in her by a cotton and after 2 sneezes, she exhibited eye-closure myotonia on both eyes lasting few minutes. There were no dystrophic changes noted and her musculoskeletal and neurological examination were normal including muscle power. No hypertrophic changes were noted in any of her skeletal muscles. She was attending her school and there were no noted difficulties with play activities. She could not recollect any seasonal variations of the disability though she presented to us in winter months and warming test were negative in her myotonic disability. She was born as the third child of consanguineous parents with no family history of similar illness. Routine biochemical investigations including serum electrolytes and creatine kinase were normal. Nerve conduction study was normal. Needle EMG (electromyography) revealed short runs of myotonic discharges in the form repetitive muscle fibre afterdischarges on tapping the muscle belly (dive-bomber phenomenon) of left biceps brachie, brachioradialis and rectus femoris. She was diagnosed to have isolated eyelid myotonia. No medication were prescribed and the parents were reassured. Further diagnostic workup including molecular genetic study was not carried out. She is on continuous follow up to assess the progress of the disease.

\section{Discussion}

Isolated eyelid myotonia is a rare myotonic disorder not reported in children and seen in adults either as component of myotonic dystrophy spectrum and/or other progressive myotonic disorder. ${ }^{4}$ They are grouped under non-dystrophic myotonias and considered as a form of skeletal muscle channelopathies, electrophysiologically characterized by altered membrane excitability. ${ }^{5,6}$ In contrast to myotonic dystrophy that is characterized by progressive muscle wasting and systemic manifestations, complaints in this condition is particularly related to eyelids only. Myotonia, can also get aggravated by exposure to cold in some of these disorders especially paramyotonias and warming the area alleviates the symptoms. ${ }^{7}$ Myotonia congenita 
is inherited in autosomal dominant or recessive manner and caused by mutations in the skeletal muscle chloride channel gene (CLCN-1). ${ }^{5}$ Paramyotonia congenita and the sodium channel myotonias are autosomal dominant disorders caused by mutations in the skeletal muscle sodium channel gene (SCN4A). ${ }^{5}$ There are 50 different types of sodium channel mutations identified in the SCN4A gene. ${ }^{1}$ The M1476I mutation is associated with severe painful myotonia and a clear warm-up phenomenon and the N1297K mutation is associated with early-onset muscle hypertrophy, psychomotor retardation and fatal outcome..$^{2,8} \mathrm{~A}$ mild phenotype is characterized by myotonia of the orbicularis oculi with a warm-up phenomenon and minimal or no myotonia elsewhere. ${ }^{4}$ Testing for eyelid closure myotonia is an important to distinguish between myotonic dystrophy (MD) and non-dystrophic myotonic syndromes (NDM). MD is characterized by weakness of the eyelid opening muscles and as compared to NDM where there is by eyelid closure myotonia. ${ }^{5}$ In our patient, there was inability to open the eyelids though she had no other muscle weakness. She is on regular follow-up to assess disease progression.

There is currently insufficient clinical evidence to recommend a standard treatment. More specific pharmacological approaches are required to preferentially reduce persisted muscle sodium currents. Target therapy to prevent premature mutant channel degradation or correction of mis-targeted mutant gene is a theoretical possibility. ${ }^{9,10}$

\section{Compliance with Ethical Standards}

Funding None

Conflict of Interest None

\section{References:}

1. Sansone VA. The Dystrophic and Nondystrophic Myotonias. Continuum (Minneap Minn). 2016;22(6, Muscle and Neuromuscular Junction Disorders):1889-1915

2. Mankodi A, Thornton CA. Myotonic syndromes. Curr Opin Neurol. 2002;15:545-552

3. Vicart S, Sternberg D, Fontaine B, Meola G. Human skeletal muscle sodium channelopathies. Neurol Sci. 2005;26:194202.

4. Stunnenberg BC, Ginjaar HB, Trip J, Faber CG, van Engelen BG, Drost G. Isolated eyelid closure myotonia in two families with sodium channel myotonia. Neurogenetics. 2010;11:257-260.

5. Trip J, Drost G, Ginjaar I, Nieman F, van der Kooi AJ, de Visser $M$ et al. Redefining the clinical phenotypes of non-dystrophic myotonic syndromes. J Neurol Neurosurg Psychiatry 2009; 80: 647-652.

6. Matthews E, Fialho D, Tan SV, Venance SL, Cannon $\mathrm{SC}$, Sternberg $\mathrm{D}$, et al; CINCH Investigators. The nondystrophic myotonias: molecular pathogenesis, diagnosis and treatment. Brain. 2010;133(Pt 1):9-22.

7. Haass A, Ricker $K$, Rüdel R, Lehmann-Horn F, Böhlen R, Dengler R, Mertens HG. Clinical study of paramyotonia congenita with and without myotonia in a warm environment. Muscle Nerve. 1981;4:388-395.

8. Caldwell JH. Clustering of sodium channels at the neuromuscular junction. Microsc Res Tech. 2000;49:84-89.

9. Harper P. Myotonic dystrophy: a multisystemic disorder. In: Harper P, Van Engelen B, Eymard B, Wilcox D. Myotonic dystrophy: present management, future therapy. Oxford University Press, Oxford, England.2004: 3-13

10. LoRusso S, Weiner B, Arnold WD. Myotonic Dystrophies: Targeting Therapies for Multisystem Disease. Neurotherapeutics. 2018;15:872-884. 